Health Informatics - An International Journal (HIIJ) Vol.2, No.1, February 2013

\title{
AN ElEMENTARY Note ON SKIN HydRATION MEASUREMENT USING MEMRISTIVE EFFECT
}

\author{
T. D. Dongale \\ School of Nanoscience and Technology, Shivaji University Kolhapur \\ E-Mail: tukaram.eln@gmail.com
}

\begin{abstract}
The Memristor was predicted by Prof. L. Chua in 1971 and first prototype was reported by team of HP researcher. The memristor follows interesting relation in the view of magnetic flux and charge. There are tremendous applications areas emerged out in the framework of memristor in last few years. The applications in the Memory Technology, Neuromorphic Hardware solution, Soft Computing are name of few. The memristor was hidden at many instances in biomedical fields, but recently reported literature revels that memristor is universal part of medical diagnosis. In the bird eye view of this scenario, this paper deals with elementary note on the skin hydration measurement using memristor.
\end{abstract}

\section{KEYWORDS}

Forth Circuit Element; Memristor; Hydration Measurement

\section{INTRODUCTION}

The memristor, world known as fourth fundamental circuit element that acquires property of pinched hysteresis loop in I-V plane and monotonically increasing nonlinear charge and flux characteristics $[1,2]$. It also possesses the frequency dependent memristance property at low frequency of operation [3]. The memristor was firstly predicted by circuit theorist Prof. Leon Chua in 1971, on the symmetric principle [1] and physically realized by HP (Hewlett-Packard) researchers under the direction of R. Stanley Williams during the year 2008 [2]. In the history few principle are also predicted using symmetry principles, e.g. the displacement current in Maxwell's equations, a positron and a magnetic monopole. The first two have been experimentally observed, while the third one remains mysterious [4]. The physical structure of memristor with its equivalent circuit model is shown in Fig. 1 [2]. The fig. 2 shows relationship between four fundamental circuit elements and also completes the missing link between charge and flux linkage.
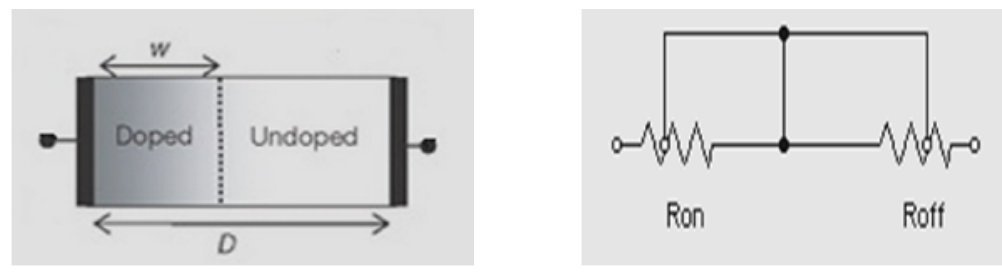

Figure 1: Structure of Memristor Reported by HP Laboratory and Its Equivalent Electrical Model. Memristor is same as that of analog variable resistor in which resistance is depends upon direction of current flow. It has of doped low resistance and undoped high resistance regions. [2] 


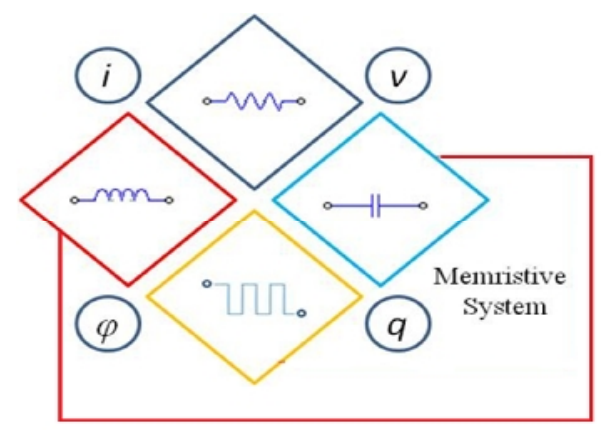

Figure 2: Relationship between Four Fundamental Circuit Elements: Resistor, Capacitor, Inductor and Memristor. A resistor is defined by the relationship between voltage (v) and current (i), the capacitor is defined by the relationship between charge (q) and voltage (v) and the inductor is defined by the relationship between flux $(\varphi)$ and current (i). Prof. Leon Chua predicted that there should be a fourth fundamental circuit element to set up the relation between charge $(q)$ and magnetic flux $(\varphi)$ without an internal power supply.

The main property of memristor is its pinched hysteresis loop in I-V plane. This pinched hysteresis loop is due to the coupled equations of motion of current-controlled memristor. These coupled equations are given by equation no. 1 and 2, [1,2]. The memristor and memristive system are related as a time dependent voltage and current and it is not mandatory to relate to magnetic fields.

$$
\begin{aligned}
v & =M(w) i \\
\frac{d q}{d t} & =i
\end{aligned}
$$

Where, $\mathrm{M}$ denotes the memristance and $\mathrm{w}$ represent the width of doped region.

The unique characteristics linear and non linear resistor with memory of memristor gives several electronic models such as, the linear ion drift model, the nonlinear ion drift model, Simmons tunnel barrier model, and the ThrEshold Adaptive Memristor (TEAM) model [1, 2, 56]. However, the memristor devices are not commercially available, good physical model-tohardware correlations have not been yet been reported in the published literature [7, 8]. The memristive class consist of the memristive $(\mathrm{MrS})$, memcapacitative $(\mathrm{McS})$ and meminductive (MiS) subsystem [7, 8]. These elements are considering as one port element whose property is depends upon the time derivative of charge and flux linkage $[4,8]$. The MATLAB based linear drift model of relation between magnetic flux $\mathrm{v} / \mathrm{s}$ charge and pinched hysteresis loop are shown in fig 3 and 4 respectively.

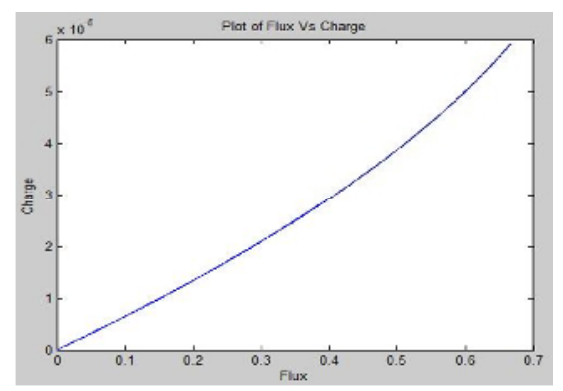

Figure 3: Simulative Plot of Flux V/S Charge at $\omega=3 \mathrm{rad} / \mathrm{s}$. The magnetic flux is a Single-valued function of charge. The monotonically increasing functions of magnetic flux $(\varphi)$ and charge $(q)$ is related as following equation, $\lim _{q \rightarrow-\infty} M(q)=$ Ron and $\lim _{q \rightarrow \infty} M(q)=$ Roff, where $0<$ Ron $<$ Roff 


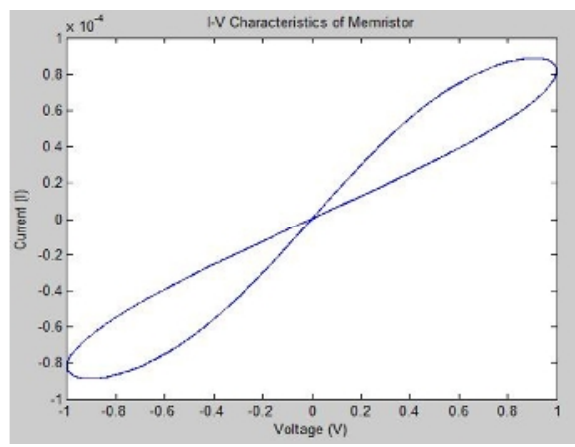

Figure 4: Simulative Plot of Current V/S Voltage at $\omega=3 \mathrm{rad} / \mathrm{s}$. The M-efficiency factor $\left(\mathrm{R}_{\mathrm{OFF}} / \mathrm{R}_{\mathrm{ON}}\right)$ is considered as 160 . The hysteresis loop represents the coupled equations of motion for current-controlled memristor (equation no. 1 and 2). The current is nonlinear with the applied voltage, resulting in hysteresis loops rather than straight lines. Theoretically, at high frequency the hysteresis loop vanishes and become a straight line.

\section{SKin Hydration MEasurement USING MeMristor}

As far as the biomedical field is concerned, there are a many observed hysteretic or nonlinear $\mathrm{i}-\mathrm{v}$ characteristics are found in the field of bioimpedance and its allied fields [3, 9-18]. The scientist Liang Li and Edward Cox at Princeton University reported that cells have a rudimentary memory, which is similar to memristor [13]. As reported [3], our skin behaves as a memristor due to the dominance of the sweat ducts on the skin conductance at sufficiently low frequencies. When negative low frequency signal is applied to the human skin, the liquid level is rises in sweat ducts due to electro-osmosis process and at the positive cycle, the liquid level is fall in sweat ducts [3]. So, it is sign of memristor behaviour in the human skin [3, 4]. The well known equivalent electrical model of the human skin addition to memristor given by G. K. Johnsen is shown in the fig. 5, [Adopted from ref. 3 (Gorm K. Johnsen, 2012)]

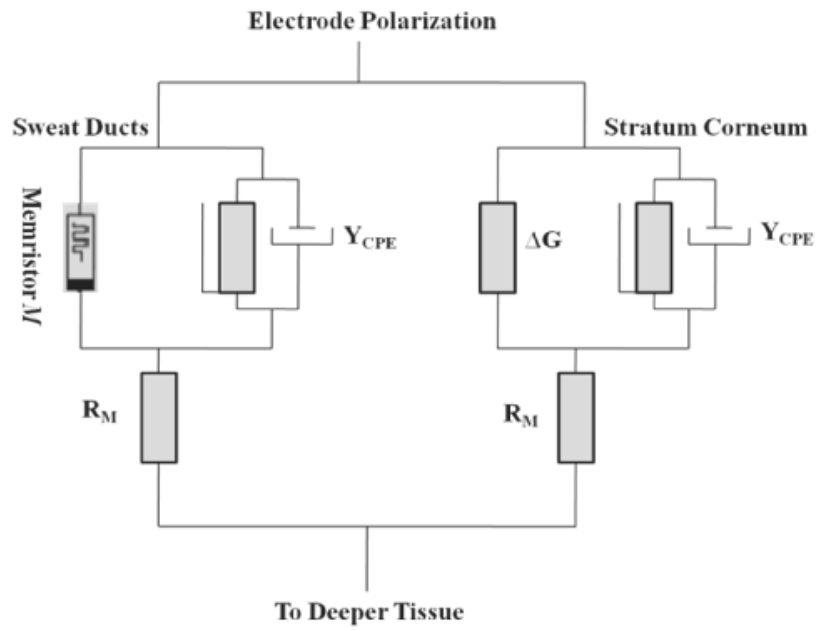

Figure 5: Equivalent Electrical Model of Human Skin with Memristor. Here the resistor is replaced by memristor, the model represent the equivalent DC current model. The current is nonlinear with the applied voltage in the memristor, resulting in hysteresis loops rather than straight lines. Theoretically, at high frequency the hysteresis loop vanishes and become a straight line. In human skin, the memristance property will be vanished at high frequency [3]. 
The commercial device level memristor is not realized and reported in the literature until now, but as logical point of view, the memristor is a next workflow in many sectors including biomedical instrumentation and its allied fields. In 2011, the S.P. Kosta and its research team reported the Human blood liquid memristor [17]. In their study they observe that, the human blood also possess the same characteristics as like memristor. In the vein of blood, skin also possesses hysteresis like characteristics at very low frequency and it is vanish at high frequency $[3,4,19]$. The existing hydration methods and criteria such as thirst, blood pressure, urine output, specific gravity, MRI, dilution etc. methods have limited accuracy [20]. If we are able to measure I-V characteristics including memristive nature of human skin, then precise measurement of skin hydration will be performed and it is go beyond the traditional RLC framework of biomedical fields.

The standard electrical methods for evaluating changes in skin hydration based on impedance, conductance and capacitance measurements are reported many of the literature [1921]. But as a point of view of memristor based skin hydration measurement, the capacitive method will be best suited for such proposed application. If we closely look at the fig 2 , then there is close relation between capacitor and memristor which form a memristive system. As reported many of the literature, the capacitance based measurement method consider the dielectric in the form of sweat liquids. So ultimately sweat ducts play an important role in measuring the skin hydration. The fig. 6 also shows the relation of memristor and skin. As the duct becomes more filled with sweat liquid, the capacitance will increases and if sweat duct filled with less sweat liquid then capacitance will decrease. This is due to simple well known capacitance formula. If we are able to calculate this minor change in capacitance at very low frequency then there is possibility of measurement of skin hydration using memristor and in general memristive system with infinite precision.
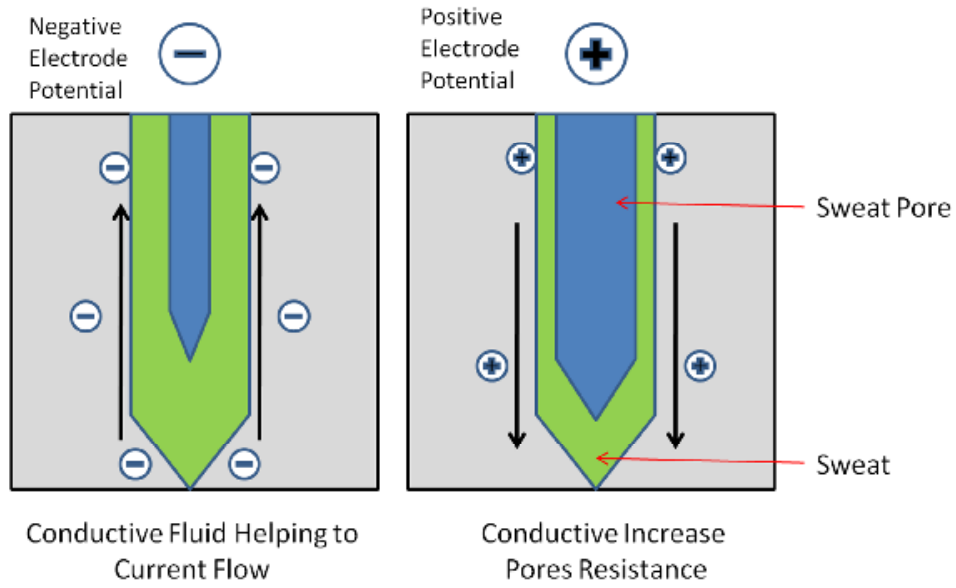

Figure 6: Cross-Sectional View of a Sweat Pores in the Skin. (Adopted from reference [22])

The proposed model for measurement of skin hydration is shown in fig. 7. The low frequency current are used for the measurement of skin hydration level with respect to memristor along with the traditional skin hydration framework. As the skin become more filled with sweat ducts due to low frequency current the capacitance will increases. If the skin becomes less filled with sweat ducts due to low frequency current the capacitance will decreases. The changes in capacitance are detected by capacitance meter. The capacitance is the sign of water filled in the skin i.e. skin hydration. Combination of conventional skin hydration measurement and memristive effect, we are able to measure précised skin hydration measurement. 


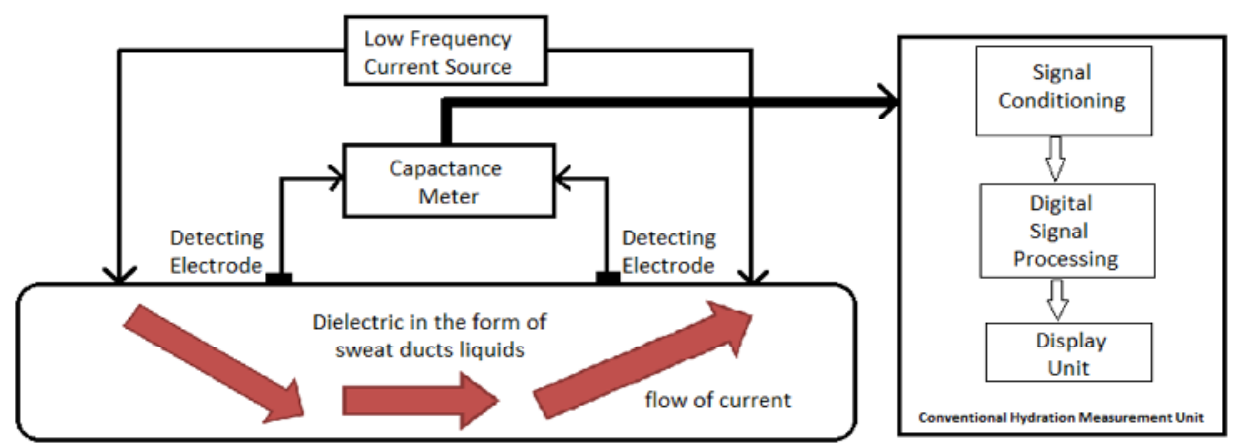

Figure 7: The Proposed Model for Measurement of Skin Hydration Using Memristive Effect. The conventional set up for hydration measurement consists of signal conditioning, digital signal processing and display unit. The conventional set up realized on FPGA or DSP board in general with low frequency memristance measurement.

\section{Conclusions}

The inclusive memristive properties of human skin make a new roadmap in the biomedical fields. The hidden memristive properties in many aspects of natural things are revels now, so ultimate precision in biomedical instrumentation are being emerged in coming future. The memristor based skin hydration is one of the areas which support precision due to memristor and traditional biomedical framework. There are many sectors regarding memristive system, where we have to look forward in coming days. The well known capacitance measurement technique is less precise, because it does not involving memristive effect in the human skin. If we are able to measure the skin hydration along with general skin hydration capacitive method then there is possibility of precise skin hydration measurement.

\section{ACKNOWLEDGEMENTS}

The author would like to thanks to the researcher over the world, those are making 'Memristor' a real-time phenomenon, especially Prof. L. Chua, R. S. Williams, Gorm. K. Johnsen and many more. The author also thanks to reviewer for their best possible suggestion regarding with this paper.

\section{REFERENCES}

[1] Chua, L. O. Memristor - the missing circuit element, IEEE Trans. Circuit Theory, 18, 1971, pp.507519.

[2] Strukov, D. B., Snider, G. S., Stewart, D. R. \& Williams, R. S. Nature, 453, 2008, pp.80-83

[3] Gorm K. Johnsen, An introduction to the memristor - a valuable circuit element in bioelectricity and bioimpedance, J Electr Bioimp, vol. 3, 2012, pp. 20 -28.

[4] Yogesh N Joglekar and Stephen J Wolf, "The elusive memristor: properties of basic electrical circuits", European Journal of Physics, vol. 30, 2009, pp. 661-675.

[5] L. Chua and S.M. Kang, "Memristive Device and Systems," Proceedings of IEEE, Vol. 64, no. 2, 1976, pp. 209-223.

[6] Z. Biolek, D. Biolek, V. Biolková, "Spice Model of Memristor with Nonlinear Dopant Drift", Radio engineering, vol. 18, no. 2, 2009, pp. 210-214.

[7] Robinson E. Pino, Kristy A. Campbell, Compact Method for Modeling and Simulation of Memristor Devices, Proceeding of international Symposium on Nanoscale Architecture, 2010, pp.1-4. 
[8] Dongale, T. D. An Overview of Fourth Fundamental Circuit Element-'The Memristor', Supporting Docs. "NanoHUB. org." Available at: https://nanohub.org/resources/16590

[9] Cole KS. Rectification and inductance in the squid giant axion, J Gen Physiol. 25; 1941; pp.29-51

[10] Cole KS, Membranes, ions, and impulses. University of California Press; Berkeley, 1972.

[11] Mauro A, Anomalous impedance, a phenomenological property of time-variant resistance - an analytic review, Biophys J. 1961; 1; pp.353-72.

[12] Johnsen GK, Lütken CA, Martinsen ØG, Grimnes S. Memristive model of electro-osmosis in skin. Phys Rev E, 83, 031916, 2011.

[13] Tiny organisms remember the way to food, Available at: http://www.newscientist.com/article/dn11394-tiny-organisms-remember-the-way-to-food.html, Retrieved: 28 December, 2012.

[14] Licht TS, Stern M, Shwachman H. Measurement of the electrical conductivity of sweat. Clin Chem. 1957;3; pp.37-48.

[15] Tronstad C, Johnsen GK, Grimnes S, Martinsen ØG. A study on electrode gels for skin conductance measurements. Physiol Meas. 2010;31;pp.1395-1410.

[16] Martinsen, Ø. G., Grimnes, S., Lütken, C. A., \& Johnsen, G. K. (2010, May). Memristance in human skin. In Journal of Physics: Conference Series (Vol. 224, No. 1, p. 012071). IOP Publishing.

[17] S.P. Kosta, Y.P. Kosta, Mukta Bhatele, Y.M. Dubey, Avinash Gaur, Shakti Kosta, Jyoti Gupta, Amit Patel and Bhavin Patel, Human blood liquid memristor, Int. J. Medical Engineering and Informatics, Vol. 3, No. 1, 2011

[18] Grimnes S, Psychogalvanic reflex and changes in electrical parameters of dry skin. Med Biol Eng Comp1982; 20; pp.734-40.

[19] Grimnes S. Skin impedance and electro-osmosis in the human epidermis. Med Biol Eng Comp. 1983;21;pp.739-49

[20] R L Gunter, W D Delinger, T L Porter, R Stewart, J Reed, Human hydration level monitoring using embedded piezoresistive microcantilever sensors, Medical Engineering \& Physics, Vol.27, issue-3, pp. $215-220$

[21] Zhang, S. L., Meyers, C. L., Subramanyan, K., \& Hancewicz, T. M. (2005). Near infrared imaging for measuring and visualizing skin hydration- A comparison with visual assessment and electrical methods, Journal of biomedical optics, 10(3), 031107-031107

[22] Memristor in human skin, Available at: http://www.newscientist.com/article/mg20928024.500sweat-ducts-make-skin-a-memristor.html, Retrieved: 28 December, 2012.

\section{Author Bibliography}

Mr. T. D. Dongale was born in 1989, India. He did his Bachelors and Masters in Electronics specialized in Embedded Systems. He is Assistant Professor in School of Nanoscience and Technology, SUK, Kolhapur. He also qualified the State Eligibility Test for Lectureship (SET) and National Eligibility Test for Lectureship with Junior Research Fellowship (NET-JRF) during his second year of Masters itself. He has been awarded 'Merit Scholarship' of the Shivaji University, Kolhapur for securing the first rank in his graduation and post graduation studies. Moreover he is also a recipient of the 'Eklavya Scholarship' for supporting his Masters studies. He has to his credit 15 research papers published in reputed international journals and author of three book 'The Treatise on sensor interfacing' (Germany, Lap- Lambert, 2012)', 'Annals of Scholarly Research in Electronic', (Germany, Lap- Lambert, 2012)', 'ZigBee and RFID Based System Design', (Germany, Lap- Lambert, 2012)'. His current research interests are Fuzzy Logic, Artificial Neural Network, Feedback Control System, Power Electronics and Memristor.

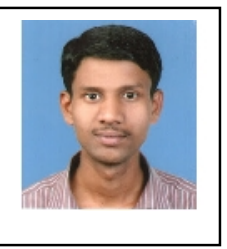

